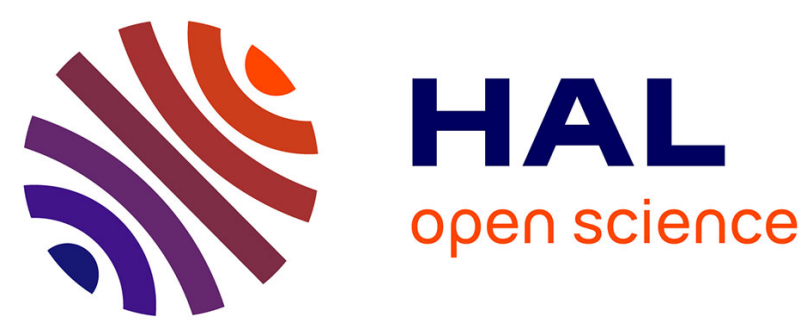

\title{
Distributed control synthesis using Euler's method
}

Adrien Le Coënt, Julien Alexandre Dit Sandretto, Alexandre Chapoutot,

Laurent Fribourg, Florian de Vuyst, Ludovic Chamoin

\section{To cite this version:}

Adrien Le Coënt, Julien Alexandre Dit Sandretto, Alexandre Chapoutot, Laurent Fribourg, Florian de Vuyst, et al.. Distributed control synthesis using Euler's method. International Workshop on Reachability Problems, Lecture Notes in Computer Science (10506), pp.118-131, 2017, 10.1007/9783-319-67089-8_9 . hal-01593225

\section{HAL Id: hal-01593225 \\ https://hal.science/hal-01593225}

Submitted on 25 Sep 2017

HAL is a multi-disciplinary open access archive for the deposit and dissemination of scientific research documents, whether they are published or not. The documents may come from teaching and research institutions in France or abroad, or from public or private research centers.
L'archive ouverte pluridisciplinaire HAL, est destinée au dépôt et à la diffusion de documents scientifiques de niveau recherche, publiés ou non, émanant des établissements d'enseignement et de recherche français ou étrangers, des laboratoires publics ou privés. 


\title{
Distributed control synthesis using Euler's method
}

\author{
Adrien Le Coënt ${ }^{*} \quad$ Julien Alexandre dit Sandretto ${ }^{\dagger}$ \\ Alexandre Chapoutot ${ }^{\dagger} \quad$ Laurent Fribourg ${ }^{\ddagger}$ \\ Florian de Vuyst* $\quad$ Ludovic Chamoin ${ }^{\S}$
}

September 25, 2017

\begin{abstract}
In a previous work, we explained how Euler's method for computing approximate solutions of systems of ordinary differential equations can be used to synthesize safety controllers for sampled switched systems. We continue here this line of research by showing how Euler's method can also be used for synthesizing safety controllers in a distributed manner. The global system is seen as an interconnection of two (or more) subsystems where, for each component, the sub-state corresponding to the other component is seen as an "input"; the method exploits (a variant of) the notions of incremental input-to-state stability $(\delta-I S S)$ and ISS Lyapunov function. We illustrate this distributed control synthesis method on a building ventilation example.
\end{abstract}

\section{Introduction}

The computation of reachable sets for continuous-time dynamical systems has been intensively studied during the last decades. Most of the methods to compute the reachable set start from an initial value problem for a system of ordinary differential equations (ODE) defined by

$$
\dot{x}(t)=f(t, x(t)) \quad \text { with } \quad x(0) \in X_{0} \subset \mathbb{R}^{n} \quad \text { and } \quad t \in\left[0, t_{\text {end }}\right] .
$$

As an analytical solution of Equation (1) is usually not computable, numerical approaches have been considered. A numerical method to solve Equation (1),

\footnotetext{
${ }^{1}$ CMLA, CNRS, ENS Paris-Saclay, INRIA

61 av. du Président Wilson, 94235 Cachan cedex, France

adrien.le-coent@ens-cachan.fr, devuyst@cmla.ens-cachan.fr

${ }^{2}$ U2IS, ENSTA ParisTech

828 bd des Maréchaux, 91762 Palaiseau, France

alexandre. chapoutot@ensta-paristech.fr,

julien.alexandre-dit-sandretto@ensta-paristech.fr

${ }^{3}$ LSV, CNRS, ENS Paris-Saclay, INRIA

61 av. du Président Wilson, 94235 Cachan cedex, France

fribourg@lsv.ens-cachan.fr

${ }^{4}$ LMT, CNRS, ENS Paris-Saclay, INRIA

61 av. du Président Wilson, 94235 Cachan cedex, France

chamoin@lmt.ens-cachan.fr
} 
when $X_{0}$ is reduced to one value, produces a discretization of time, such that $t_{0} \leqslant \cdots \leqslant t_{N}=t_{\text {end }}$, and a sequence of states $x_{0}, \ldots, x_{N}$ based on an integration method which starts from an initial value $x_{0}$ at time $t_{0}$ and a finite time horizon $h$ (the step-size), produces an approximation $x_{k+1}$ at time $t_{k+1}=t_{k}+h$, of the exact solution $x\left(t_{k+1}\right)$, for all $k=0, \ldots, N-1$. The simplest numerical method is Euler's method in which $t_{k+1}=t_{k}+h$ for some step-size $h$ and $x_{k+1}=x_{k}+h f\left(t_{k}, x_{k}\right)$; so the derivative of $x$ at time $t_{k}, f\left(t_{k}, x_{k}\right)$, is used as an approximation of the derivative on the whole time interval.

The global error error $(t)$ at $t=t_{0}+k h$ is equal to $\left\|x(t)-x_{k}\right\|$. In case $n=1$, if the solution $x$ has a bounded second derivative and $f$ is Lipschitz continuous in its second argument, then it satisfies:

$$
\operatorname{error}(t) \leq \frac{h M}{2 L}\left(e^{L\left(t-t_{0}\right)}-1\right)
$$

where $M$ is an upper bound on the second derivative of $x$ on the given interval and $L$ is the Lipschitz constant of $f[3]{ }^{1}$

In [13], we gave an upper bound on the global error error $(t)$, which is more precise than (2). This upper bound makes use of the notion of One-Sided Lipschitz (OSL) constant. This notion has been used for the first time by [7] in order to treat "stiff" systems of differential equations for which the explicit Euler method is numerically "unstable" (unless the step size is taken to be extremely small). Unlike Lipschitz constants, OSL constants can be negative, which express a form of contractivity of the system dynamics. Even if the OSL constant is positive, it is in practice much lower than the Lipschitz constant [5]. The use of OSL thus allows us to obtain a much more precise upper bound for the global error. We also explained in [13] how such a precise estimation of the global error can be used to synthesize safety controllers for a special form hybrid systems, called "sampled switched systems".

In this paper, we explain how such an Euler-based method can be extended to synthesize safety controllers in a distributed manner. This allows us to control separately a component using only partial information on the other components. It also allows us to scale up the size of the global systems for which a control can be synthesized. In order to perform such a distributed synthesis, we will see the components of the global systems as being interconnected (see, e.g., [17]), and use (a variant of) the notions of incremental input-to-state stability $(\delta$-ISS) and ISS Lyapunov functions [10] instead of the notion of OSL used in the centralized framework.

The plan of the paper is as follows: In Section 2, we recall the results of [13] obtained in the centralized framework; in Section 3 we extend these results to the framework of distributed systems; we then apply the distributed synthesis method to a nontrivial example (Section 4), and conclude in Section 5.

\footnotetext{
${ }^{1}$ Such a bound has been used in hybridization methods: $\operatorname{error}(t)=\frac{E_{D}}{L}\left(e^{L t}-1\right)[2,4]$, where $E_{D}$ gives the maximum difference of the derivatives of the original and approximated systems.
} 


\section{Euler's method applied to control synthesis}

In this Section, we recall the results obtained in [13]. We first give results concerning a system governed by a single ODE system (Section 2.1), then consider results for a switched system composed of several ODEs (Section 2.2).

\subsection{ODE systems}

We make the following hypothesis:

(H0) $\quad f$ is a locally Lipschitz continuous map.

We make the assumption that the vector field $f$ is such that the solutions of the differential equation (7) are defined. We will denote by $\phi\left(t ; x^{0}\right)$ the solution at time $t$ of the system:

$$
\begin{aligned}
\dot{x}(t) & =f(x(t)), \\
x(0) & =x^{0} .
\end{aligned}
$$

Consider a compact and convex set $S \subset \mathbb{R}^{n}$, called "safety set". We denote by $T$ a compact overapproximation of the image by $\phi$ of $S$ for $0 \leq t \leq \tau$, i.e., $T$ is such that

$$
T \supseteq\left\{\phi\left(t ; x^{0}\right) \mid 0 \leq t \leq \tau, x^{0} \in S\right\} .
$$

The existence of $T$ is guaranteed by assumption (H0). We know furthermore by $(H 0)$ that there exists a constant $L>0$ such that:

$$
\|f(y)-f(x)\| \leq L\|y-x\| \quad \forall x, y \in S .
$$

Let us define $C$ :

$$
C=\sup _{x \in S} L\|f(x)\| .
$$

We make the additional hypothesis that the mapping $f$ is one-sided Lipschitz (OSL) [7]. Formally:

(H1) There exists a constant $\lambda \in \mathbb{R}$ such that

$$
\langle f(y)-f(x), y-x\rangle \leq \lambda\|y-x\|^{2} \quad \forall x, y \in T,
$$

where $\langle\cdot, \cdot\rangle$ denotes the scalar product of two vectors of $\mathbb{R}^{n}$.

Remark 1. Constants $\lambda, L$ and $C$ can be computed using (constrained) optimization algorithms.

Given an initial point $\tilde{x}^{0} \in S$, we define the following "linear approximate solution" $\tilde{\phi}\left(t ; \tilde{x}^{0}\right)$ for $t$ on $[0, \tau]$ by:

$$
\tilde{\phi}\left(t ; \tilde{x}^{0}\right)=\tilde{x}^{0}+t f\left(\tilde{x}^{0}\right) .
$$

We define the closed ball of center $x \in \mathbb{R}^{n}$ and radius $r>0$, denoted $B(x, r)$, as the set $\left\{x^{\prime} \in \mathbb{R}^{n} \mid\left\|x^{\prime}-x\right\| \leq r\right\}$.

Given a positive real $\delta$, we now define the expression $\delta(t)$ which, as we will see in Theorem 1, represents (an upper bound on) the error associated to $\tilde{\phi}\left(t ; \tilde{x}^{0}\right)$ (i.e., $\left.\left\|\tilde{\phi}\left(t ; \tilde{x}^{0}\right)-\phi\left(t ; x^{0}\right)\right\|\right)$. 


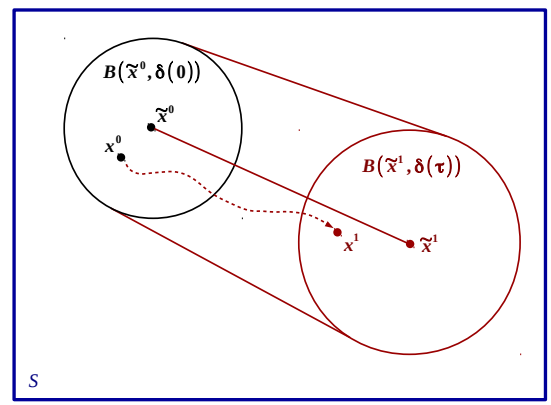

Figure 1: Illustration of Corollary 1, with $\tilde{x}_{1}=\tilde{\phi}\left(\tau ; \tilde{x}^{0}\right)$ and $x_{1}=\phi\left(\tau ; x^{0}\right)$.

Definition 1. Let $\delta$ be a positive constant. Let us define, for all $0 \leq t \leq \tau, \delta(t)$ as follows:

- if $\lambda<0$ :

$$
\delta(t)=\left(\delta^{2} e^{\lambda t}+\frac{C^{2}}{\lambda^{2}}\left(t^{2}+\frac{2 t}{\lambda}+\frac{2}{\lambda^{2}}\left(1-e^{\lambda t}\right)\right)\right)^{\frac{1}{2}}
$$

- if $\lambda=0$ :

$$
\delta(t)=\left(\delta^{2} e^{t}+C^{2}\left(-t^{2}-2 t+2\left(e^{t}-1\right)\right)\right)^{\frac{1}{2}}
$$

- if $\lambda>0$ :

$$
\delta(t)=\left(\delta^{2} e^{3 \lambda t}+\frac{C^{2}}{3 \lambda^{2}}\left(-t^{2}-\frac{2 t}{3 \lambda}+\frac{2}{9 \lambda^{2}}\left(e^{3 \lambda t}-1\right)\right)\right)^{\frac{1}{2}}
$$

Note that $\delta(t)=\delta$ for $t=0$. The function $\delta(\cdot)$ depends implicitly on parameter: $\delta \in \mathbb{R}$. In Section 2.2, we will use the notation $\delta^{\prime}(\cdot)$ where the parameter is denoted by $\delta^{\prime}$.

Theorem 1. Given an ODE system satisfying (H0-H1), consider a point $\tilde{x}^{0}$ and a positive real $\delta$. We have, for all $x^{0} \in B\left(\tilde{x}^{0}, \delta\right), t \in[0, \tau]$ :

$$
\phi\left(t ; x^{0}\right) \in B\left(\tilde{\phi}\left(t ; \tilde{x}^{0}\right), \delta(t)\right) .
$$

Corollary 1. Given an ODE system satisfying (HO-H1), consider a point $\tilde{x}^{0} \in$ $S$ and a real $\delta>0$ such that:

1. $B\left(\tilde{x}^{0}, \delta\right) \subseteq S$,

2. $B\left(\tilde{\phi}\left(\tau ; \tilde{x}^{0}\right), \delta(\tau)\right) \subseteq S$, and

3. $\frac{d^{2}(\delta(t))}{d t^{2}}>0$ for all $t \in[0, \tau]$.

Then we have, for all $x^{0} \in B\left(\tilde{x}^{0}, \delta\right)$ and $t \in[0, \tau]: \phi\left(t ; x^{0}\right) \in S$. 


\subsection{Sampled switched systems}

Let us consider the nonlinear switched system

$$
\dot{x}(t)=f_{\sigma(t)}(x(t))
$$

defined for all $t \geq 0$, where $x(t) \in \mathbb{R}^{n}$ is the state of the system, $\sigma(\cdot): \mathbb{R}^{+} \longrightarrow U$ is the switching rule. The finite set $U=\{1, \ldots, N\}$ is the set of switching modes of the system. We focus on sampled switched systems: given a sampling period $\tau>0$, switchings will occur at times $\tau, 2 \tau, \ldots$ The switching rule $\sigma(\cdot)$ is thus constant on the time interval $[(k-1) \tau, k \tau)$ for $k \geq 1$. For all $j \in U, f_{j}$ is a function from $\mathbb{R}^{n}$ to $\mathbb{R}^{n}$.

We will denote by $\phi_{\sigma}\left(t ; x^{0}\right)$ the solution at time $t$ of the system:

$$
\begin{aligned}
\dot{x}(t) & =f_{\sigma(t)}(x(t)), \\
x(0) & =x^{0} .
\end{aligned}
$$

Often, we will consider $\phi_{\sigma}\left(t ; x^{0}\right)$ on the interval $0 \leq t<\tau$ for which $\sigma(t)$ is equal to a constant, say $j \in U$. In this case, we will abbreviate $\phi_{\sigma}\left(t ; x^{0}\right)$ as $\phi_{j}\left(t ; x^{0}\right)$. We will also consider $\phi_{\sigma}\left(t ; x^{0}\right)$ on the interval $0 \leq t<k \tau$ where $k$ is a positive integer, and $\sigma(t)$ is equal to a constant, say $j_{k^{\prime}}$, on each interval $\left[\left(k^{\prime}-1\right) \tau, k^{\prime} \tau\right)$ with $1 \leq k^{\prime} \leq k$; in this case, we will abbreviate $\phi_{\sigma}\left(t ; x^{0}\right)$ as $\phi_{\pi}\left(t ; x^{0}\right)$, where $\pi$ is a sequence of $k$ modes (or "pattern") of the form $\pi=$ $j_{1} \cdot j_{2} \cdots j_{k}$.

We will assume that $\phi_{\sigma}$ is continuous at time $k \tau$ for all positive integer $k$. This means that there is no "reset" at time $k^{\prime} \tau\left(1 \leq k^{\prime} \leq k\right)$; the value of $\phi_{\sigma}\left(t, x^{0}\right)$ for $t \in\left[\left(k^{\prime}-1\right) \tau, k \tau\right]$ corresponds to the solution of $\dot{x}(u)=f_{j_{k^{\prime}}}(x(u))$ for $u \in[0, \tau]$ with initial value $\phi_{\sigma}\left(\left(k^{\prime}-1\right) \tau ; x^{0}\right)$.

More generally, given an initial point $\tilde{x}^{0} \in S$ and pattern $\pi$ of $U^{k}$, we can define a "(piecewise linear) approximate solution" $\tilde{\phi}_{\pi}\left(t ; \tilde{x}^{0}\right)$ of $\phi_{\pi}$ at time $t \in[0, k \tau]$ as follows:

- $\tilde{\phi}_{\pi}\left(t ; \tilde{x}^{0}\right)=t f_{j}\left(\tilde{x}^{0}\right)+\tilde{x}^{0}$ if $\pi=j \in U, k=1$ and $t \in[0, \tau]$, and

- $\tilde{\phi}_{\pi}\left(k \tau+t ; \tilde{x}^{0}\right)=t f_{j}(\tilde{z})+\tilde{z}$ with $\tilde{z}=\tilde{\phi}_{\pi^{\prime}}\left((k-1) \tau ; \tilde{x}^{0}\right)$, if $k \geq 2, t \in[0, \tau]$, $\pi=j \cdot \pi^{\prime}$ for some $j \in U$ and $\pi^{\prime} \in U^{k-1}$.

We wish to synthesize a safety control $\sigma$ for $\phi_{\sigma}$ using the approximate functions $\tilde{\phi}_{\pi}$. Hypotheses (H0) and (H1), as defined in Section 2.1, are naturally extended to every mode $j$ of $U$, as well as definition of $T$, constants $L, C$ and $\lambda$, definitions of $\tilde{\phi}_{j}$ and $\delta$ (see [13]). From a notation point of view, we will assign an index $j$ to symbols $\lambda, L, C, \ldots$ in order to relate them to the dynamics of mode $j$.

Consider a point $\tilde{x}^{0} \in S$, a positive real $\delta$ and a pattern $\pi$ of length $k$. Let $\pi\left(k^{\prime}\right)$ denote the $k^{\prime}$-th element (mode) of $\pi$ for $1 \leq k^{\prime} \leq k$. Let us abbreviate the $k^{\prime}$-th approximate point $\tilde{\phi}_{\pi}\left(k^{\prime} \tau ; \tilde{x}^{0}\right)$ as $\tilde{x}_{\pi}^{k^{\prime}}$ for $\bar{k}^{\prime}=1, \ldots, k$, and let $\tilde{x}_{\pi}^{k^{\prime}}=\tilde{x}^{0}$ for $k^{\prime}=0$. It is easy to show that $\tilde{x}_{\pi}^{k^{\prime}}$ can be defined recursively for $k^{\prime}=1, \ldots, k$, by: $\tilde{x}_{\pi}^{k^{\prime}}=\tilde{x}_{\pi}^{k^{\prime}-1}+\tau f_{j}\left(\tilde{x}_{\pi}^{k^{\prime}-1}\right)$ with $j=\pi\left(k^{\prime}\right)$.

Let us now define the expression $\delta_{\pi}^{k^{\prime}}$ as follows: For $k^{\prime}=0: \delta_{\pi}^{k^{\prime}}=\delta$, and for $1 \leq k^{\prime} \leq k: \delta_{\pi}^{k^{\prime}}=\delta_{j}^{\prime}(\tau)$ where $\delta^{\prime}$ denotes $\delta_{\pi}^{k^{\prime}-1}$, and $j$ denotes $\pi\left(k^{\prime}\right)$. Likewise, for $0 \leq t \leq k \tau$, let us define the expression $\delta_{\pi}(t)$ as follows: 
- for $t=0: \delta_{\pi}(t)=\delta$,

- for $0<t \leq k \tau: \delta_{\pi}(t)=\delta_{j}^{\prime}\left(t^{\prime}\right)$ with $\delta^{\prime}=\delta_{\pi}^{\ell-1}, j=\pi(\ell), t^{\prime}=t-(\ell-1) \tau$ and $\ell=\left\lceil\frac{t}{\tau}\right\rceil$.

Note that, for $0 \leq k^{\prime} \leq k$, we have: $\delta_{\pi}\left(k^{\prime} \tau\right)=\delta_{\pi}^{k^{\prime}}$. We have

Theorem 2. Given a sampled switched system satisfying (H0-H1), consider a point $\tilde{x}^{0} \in S$, a positive real $\delta$ and a pattern $\pi$ of length $k$ such that, for all $1 \leq k^{\prime} \leq k$ :

1. $B\left(\tilde{x}_{\pi}^{k^{\prime}}, \delta_{\pi}^{k^{\prime}}\right) \subseteq S$ and

2. $\frac{d^{2}\left(\delta_{j}^{\prime}(t)\right)}{d t^{2}}>0$ for all $t \in[0, \tau]$, with $j=\pi\left(k^{\prime}\right)$ and $\delta^{\prime}=\delta_{\pi}^{k^{\prime}-1}$.

Then we have, for all $x^{0} \in B\left(\tilde{x}^{0}, \delta\right)$ and $t \in[0, k \tau]: \phi_{\pi}\left(t ; x^{0}\right) \in S$.

Remark 2. In Theorem 2, we have supposed that the step size $h$ used in Euler's method was equal to the sampling period $\tau$ of the switching system. Actually, in order to have better approximations, it is often convenient to take a fraction of $\tau$ as for $h\left(e . g ., h=\frac{\tau}{10}\right)$. Such a splitting is called "sub-sampling" in numerical methods.

Consider now a compact set $R$, called "recurrence set", contained in the safety set $S \subset \mathbb{R}^{n}(R \subseteq S)$. We are interested in the synthesis of a control such that: starting from any initial point $x \in R$, the controlled trajectory always returns to $R$ within a bounded time while never leaving $S$.

Corollary 2. Given a switched system satisfying (HO-H1), consider a positive real $\delta$ and a finite set of points $\tilde{x}_{1}, \ldots \tilde{x}_{m}$ of $S$ such that all the balls $B\left(\tilde{x}_{i}, \delta\right)$ cover $R$ and are included into $S$ (i.e., $R \subseteq \bigcup_{i=1}^{m} B\left(\tilde{x}_{i}, \delta\right) \subseteq S$ ).

Suppose furthermore that, for all $1 \leq i \leq m$, there exists a pattern $\pi_{i}$ of length $k_{i}$ such that:

1. $B\left(\left(\tilde{x}_{i}\right)_{\pi_{i}}^{k^{\prime}}, \delta_{\pi_{i}}^{k^{\prime}}\right) \subseteq S$, for all $k^{\prime}=1, \ldots, k_{i}-1$

2. $B\left(\left(\tilde{x}_{i}\right)_{\pi_{i}}^{k_{i}}, \delta_{\pi_{i}}^{k_{i}}\right) \subseteq R$.

3. $\frac{d^{2}\left(\delta_{j}^{\prime}(t)\right)}{d t^{2}}>0$ with $j=\pi_{i}\left(k^{\prime}\right)$ and $\delta^{\prime}=\delta_{\pi_{i}}^{k^{\prime}-1}$, for all $k^{\prime} \in\left\{1, \ldots, k_{i}\right\}$ and $t \in[0, \tau]$.

These properties induce a control $\sigma^{2}$ which guarantees

- (safety): if $x \in R$, then $\phi_{\sigma}(t ; x) \in S$ for all $t \geq 0$, and

- (recurrence): if $x \in R$ then $\phi_{\sigma}(k \tau ; x) \in R$ for some $k \in\left\{k_{1}, \ldots, k_{m}\right\}$.

Corollary 2 gives the theoretical foundations of the following method for synthesizing $\sigma$ ensuring recurrence in $R$ and safety in $S$ :

\footnotetext{
${ }^{2}$ Given an initial point $x \in R$, the induced control $\sigma$ corresponds to a sequence of patterns $\pi_{i_{1}}, \pi_{i_{2}}, \ldots$ defined as follows: Since $x \in R$, there exists a a point $\tilde{x}_{i_{1}}$ with $1 \leq i_{1} \leq m$ such that $x \in B\left(\tilde{x}_{i_{1}}, \delta\right)$; then using pattern $\pi_{i_{1}}$, one has: $\phi_{\pi_{i_{1}}}\left(k_{i_{1}} \tau ; x\right) \in R$. Let $x^{\prime}=\phi_{\pi_{i_{1}}}\left(k_{i_{1}} \tau ; x\right)$; there exists a point $\tilde{x}_{i_{2}}$ with $1 \leq i_{2} \leq m$ such that $x^{\prime} \in B\left(\tilde{x}_{i_{2}}, \delta\right)$, etc.
} 


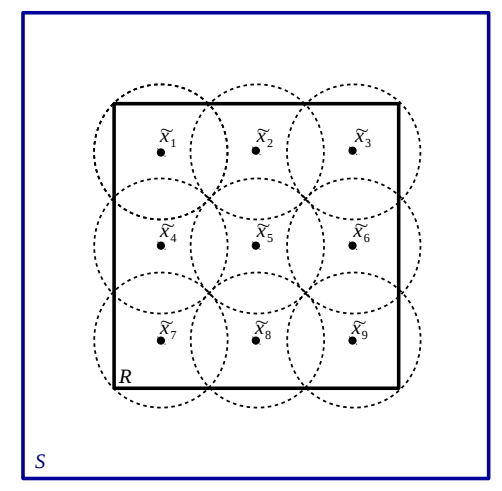

(a)

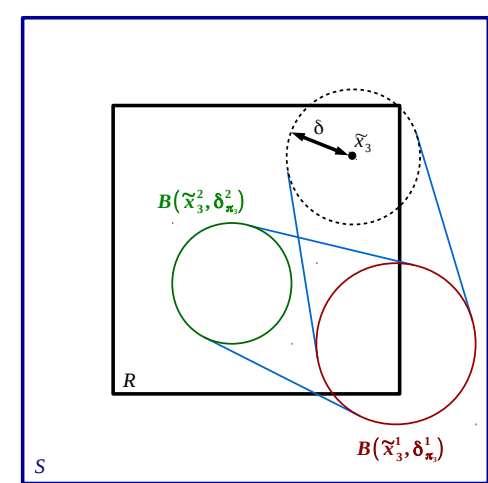

(b)

Figure 2: (a): A set of balls covering $R$ and contained in $S$. (b): Control of ball $B\left(\tilde{x}_{3}, \delta\right)$ with Euler-based method.

- we (pre-)compute $\lambda_{j}, L_{j}, C_{j}$ for all $j \in U$;

- we find $m$ points $\tilde{x}_{1}, \ldots \tilde{x}_{m}$ of $S$ and $\delta>0$ such that $R \subseteq \bigcup_{i=1}^{m} B\left(\tilde{x}_{i}, \delta\right) \subseteq$ $S$;

- we find $m$ patterns $\pi_{i}(i=1, \ldots, m)$ such that conditions 1-2-3 of Corollary 2 are satisfied.

A covering of $R$ with balls as stated in Corollary 2 is illustrated in Figure 2 (a). The control synthesis method based on Corollary 2 is illustrated in Figure 2 (b).

\section{Distributed synthesis}

The goal is to split the system into two (or more) sub-systems and synthesize controllers for the sub-systems independently. The allows to break the exponential complexity (curse of dimensionality) of the method w.r.t. the dimension of the system, as well as the dimension of the control input.

We consider the distributed control system

$$
\begin{aligned}
& \dot{x}_{1}=f_{\sigma_{1}}^{1}\left(x_{1}, x_{2}\right) \\
& \dot{x}_{2}=f_{\sigma_{2}}^{2}\left(x_{1}, x_{2}\right)
\end{aligned}
$$

where $x_{1} \in \mathbb{R}^{n_{1}}$ and $x_{2} \in \mathbb{R}^{n_{2}}$, with $n_{1}+n_{2}=n$. Furthermore, $\sigma_{1} \in U_{1}$ and $\sigma_{2} \in U_{2}$ and $U=U_{1} \times U_{2}$.

Note that the system (9-10) can be seen as the interconnection of subsystem (9) where $x_{2}$ plays the role of an "input" given by (10), with subsystem (10) where $x_{1}$ is an "input" given by (9).

Let $R=R_{1} \times R_{2}, S=S_{1} \times S_{2}, T=T_{1} \times T_{2}$ and $x_{1}^{m}$ (resp. $x_{2}^{m}$ ) be the center of $R_{1}$ (resp. $R_{2}$ ). We denote by $L_{\sigma_{1}}^{1}$ the Lipschitz constant for sub-system 1 under mode $\sigma_{1}$ :

$$
\left\|f_{\sigma_{1}}^{1}\left(x_{1}, x_{2}\right)-f_{\sigma_{1}}^{1}\left(y_{1}, y_{2}\right)\right\| \leq L_{\sigma_{1}}^{1}\left\|\left(\begin{array}{l}
x_{1} \\
x_{2}
\end{array}\right)-\left(\begin{array}{l}
y_{1} \\
y_{2}
\end{array}\right)\right\|
$$


We then introduce the constant:

$$
C_{\sigma_{1}}^{1}=\sup _{x_{1} \in S_{1}} L_{\sigma_{1}}^{1}\left\|f_{\sigma_{1}}^{1}\left(x_{1}, x_{2}^{m}\right)\right\|
$$

Similarly, we define the constants for sub-system 2:

$$
\left\|f_{\sigma_{2}}^{2}\left(x_{1}, x_{2}\right)-f_{\sigma_{2}}^{2}\left(y_{1}, y_{2}\right)\right\| \leq L_{\sigma_{2}}^{2}\left\|\left(\begin{array}{l}
x_{1} \\
x_{2}
\end{array}\right)-\left(\begin{array}{l}
y_{1} \\
y_{2}
\end{array}\right)\right\|
$$

and

$$
C_{\sigma_{2}}^{2}=\sup _{x_{2} \in S_{2}} L_{\sigma_{2}}^{2}\left\|f_{\sigma_{2}}^{2}\left(x_{1}^{m}, x_{2}\right)\right\|
$$

Let us now make additional assumptions on the coupled sub-systems, closely related to the notion of (incremental) input-to-state stability.

(H2) For every mode $\sigma_{1} \in U_{1}$, there exists constants $\lambda_{\sigma_{1}}^{1} \in \mathbb{R}$ and $\gamma_{\sigma_{1}}^{1} \in \mathbb{R}_{>0}$ such that $\forall x, x^{\prime} \in T_{1}^{2}$ and $\forall y, y^{\prime} \in T_{2}^{2}$, the following expression holds

$$
\left\langle f_{\sigma_{1}}^{1}(x, y)-f_{\sigma_{1}}^{1}\left(x^{\prime}, y^{\prime}\right), x-x^{\prime}\right\rangle \leq \lambda_{\sigma_{1}}^{1}\left\|x-x^{\prime}\right\|^{2}+\gamma_{\sigma_{1}}^{1}\left\|x-x^{\prime}\right\|\left\|y-y^{\prime}\right\| .
$$

(H3) For every mode $\sigma_{2} \in U_{2}$, there exists constants $\lambda_{\sigma_{2}}^{2} \in \mathbb{R}$ and $\gamma_{\sigma_{2}}^{2} \in \mathbb{R}_{>0}$ such that $\forall x, x^{\prime} \in T_{1}^{2}$ and $\forall y, y^{\prime} \in T_{2}^{2}$, the following expression holds

$$
\left\langle f_{\sigma_{2}}^{2}(x, y)-f_{\sigma_{2}}^{2}\left(x^{\prime}, y^{\prime}\right), y-y^{\prime}\right\rangle \leq \lambda_{\sigma_{2}}^{2}\left\|y-y^{\prime}\right\|^{2}+\gamma_{\sigma_{2}}^{2}\left\|x-x^{\prime}\right\|\left\|y-y^{\prime}\right\| .
$$

These assumptions express (a variant of) the fact that the function $V\left(x, x^{\prime}\right)=$ $\left\|x-x^{\prime}\right\|^{2}$ is an ISS-Lyapunov function (see, e.g., $[1,8]$ ). Note that all the constants defined above can be numerically computed using constrained optimization algorithms.

Let us define the distributed Euler scheme:

$$
\begin{aligned}
& \tilde{x}_{1}(\tau)=\tilde{x}_{1}(0)+\tau f_{\sigma_{1}}^{1}\left(\tilde{x}_{1}(0), x_{2}^{m}\right) \\
& \tilde{x}_{2}(\tau)=\tilde{x}_{2}(0)+\tau f_{\sigma_{2}}^{2}\left(x_{1}^{m}, \tilde{x}_{2}(0)\right)
\end{aligned}
$$

The exact trajectory is now denoted, for all $t \in[0, \tau]$, by $\phi_{\left(j_{1}, j_{2}\right)}\left(t ; x^{0}\right)$ for an initial condition $x^{0}=\left(\begin{array}{ll}x_{1}^{0} & x_{2}^{0}\end{array}\right)^{T}$, and when sub-system 1 is in mode $j_{1} \in U_{1}$, and sub-system 2 is in mode $j_{2} \in U_{2}$.

We define the approximate trajectory computed with the distributed Euler scheme by $\tilde{\phi}_{j_{1}}^{1}\left(t ; \tilde{x}_{1}^{0}\right)=\tilde{x}_{1}^{0}+t f_{\sigma_{1}}^{1}\left(\tilde{x}_{1}^{0}, x_{2}^{m}\right)$ for $t \in[0, \tau]$, when sub-system 1 is in mode $j_{1}$ and with an initial condition $\tilde{x}_{1}^{0}$. Similarly, for sub-system 2, $\tilde{\phi}_{j_{2}}^{2}\left(t ; \tilde{x}_{2}^{0}\right)=\tilde{x}_{2}^{0}+t f_{\sigma_{2}}^{2}\left(x_{1}^{m}, \tilde{x}_{2}^{0}\right)$ when sub-system 2 is in mode $j_{2}$ and with an initial condition $\tilde{x}_{2}^{0}$.

We now give a distributed version of Theorem 1 .

Theorem 3. Given a distributed sampled switched system, suppose that subsystem 1 satisfies (H2), and consider a point $\tilde{x}_{1}^{0}$ and a positive real $\delta$. We have, for all $x_{1}^{0} \in B\left(\tilde{x}_{1}^{0}, \delta\right), x_{2}^{0} \in S_{2}, t \in[0, \tau], j_{1} \in U_{1}$ and any $\sigma_{2} \in U_{2}$ :

$$
\phi_{\left(j_{1}, \sigma_{2}\right)}\left(t ; x^{0}\right)_{\mid 1} \in B\left(\tilde{\phi}_{j_{1}}^{1}\left(t ; \tilde{x}_{1}^{0}\right), \delta_{j_{1}}(t)\right) .
$$

with $x^{0}=\left(\begin{array}{ll}x_{1}^{0} & x_{2}^{0}\end{array}\right)^{T}$ and 
- if $\lambda_{j_{1}}^{1}<0$,

$$
\begin{aligned}
\delta_{j_{1}}(t)=\left(\frac{\left(C_{j_{1}}^{1}\right)^{2}}{-\left(\lambda_{j_{1}}^{1}\right)^{4}}\left(-\left(\lambda_{j_{1}}^{1}\right)^{2} t^{2}-2 \lambda_{j_{1}}^{1} t+2 e^{\lambda_{j_{1}}^{1} t}-2\right)\right. \\
\quad+\frac{1}{\left(\lambda_{j_{1}}^{1}\right)^{2}}\left(\frac{C_{j_{1}}^{1} \gamma_{j_{1}}^{1}\left|T_{2}\right|}{-\lambda_{j_{1}}^{1}}\left(-\lambda_{j_{1}}^{1} t+e^{\lambda_{j_{1}}^{1} t}-1\right)\right. \\
\left.\left.+\lambda_{j_{1}}^{1}\left(\frac{\left(\gamma_{j_{1}}^{1}\right)^{2}\left(\left|T_{2}\right| / 2\right)^{2}}{-\lambda_{j_{1}}^{1}}\left(e^{\lambda_{j_{1}}^{1} t}-1\right)+\lambda_{j_{1}}^{1} \delta^{2} e^{\lambda_{j_{1}}^{1} t}\right)\right)\right)^{1 / 2}
\end{aligned}
$$

- if $\lambda_{j_{1}}^{1}>0$,

$$
\begin{aligned}
\delta_{j_{1}}(t)= & \frac{1}{\left(3 \lambda_{j_{1}}^{1}\right)^{3 / 2}}\left(\frac{C_{1}^{2}}{\lambda_{j_{1}}^{1}}\left(-9\left(\lambda_{j_{1}}^{1}\right)^{2} t^{2}-6 \lambda_{j_{1}}^{1} t+2 e^{3 \lambda_{j_{1}}^{1} t}-2\right)\right. \\
& +3 \lambda_{j_{1}}^{1}\left(\frac{C_{1} \gamma_{j_{1}}^{1}\left|T_{2}\right|}{\lambda_{j_{1}}^{1}}\left(-3 \lambda_{j_{1}}^{1} t+e^{3 \lambda_{j_{1}}^{1} t}-1\right)\right. \\
& \left.\left.+3 \lambda_{j_{1}}^{1}\left(\frac{\left(\gamma_{j_{1}}^{1}\right)^{2}\left(\left|T_{2}\right| / 2\right)^{2}}{\lambda_{j_{1}}^{1}}\left(e^{3 \lambda_{j_{1}}^{1} t}-1\right)+3 \lambda_{j_{1}}^{1} \delta^{2} e^{3 \lambda_{j_{1}}^{1} t}\right)\right)\right)^{1 / 2}
\end{aligned}
$$

- if $\lambda_{j_{1}}^{1}=0$,

$$
\begin{aligned}
\delta_{j_{1}}(t)=\left(( C _ { j _ { 1 } } ^ { 1 } ) ^ { 2 } \left(-t^{2}-2 t\right.\right. & \left.+2 e^{t}-2\right) \\
+ & \left(C_{j_{1}}^{1} \gamma_{j_{1}}^{1}\left|T_{2}\right|\left(-t+e^{t}-1\right)\right. \\
+ & \left.\left.\left(\left(\gamma_{j_{1}}^{1}\right)^{2}\left(\left|T_{2}\right| / 2\right)^{2}\left(e^{t}-1\right)+\delta^{2} e^{t}\right)\right)\right)^{1 / 2}
\end{aligned}
$$

A similar result can be established for sub-system 2, permitting to perform a distributed control synthesis.

Proof. In order to simplify the reading, we omit the mode $j_{1}$ (which does not intervene in the proof as long as $t \in[0, \tau])$ and write the proof for $f_{j_{1}}^{1}=f_{1}$, $L_{j_{1}}^{1}=L_{1}, C_{j_{1}}^{1}=C_{1}, \lambda_{j_{1}}^{1}=\lambda_{1}$. We have

$$
\begin{aligned}
& \frac{1}{2} \frac{d\left(\left\|x_{1}-\tilde{x}_{1}\right\|^{2}\right)}{d t}=\left\langle f_{1}\left(x_{1}, x_{2}\right)-f_{1}\left(\tilde{x}_{1}(0), x_{2}^{m}\right), x_{1}-\tilde{x}_{1}\right\rangle \\
& =\left\langle f_{1}\left(x_{1}, x_{2}\right)-f_{1}\left(\tilde{x}_{1}, x_{2}^{m}\right)+f_{1}\left(\tilde{x}_{1}, x_{2}^{m}\right)-f_{1}\left(\tilde{x}_{1}(0), x_{2}^{m}\right), x_{1}-\tilde{x}_{1}\right\rangle \\
& \leq\left\langle f_{1}\left(x_{1}, x_{2}\right)-f_{1}\left(\tilde{x}_{1}, x_{2}^{m}\right), x_{1}-\tilde{x}_{1}\right\rangle+\left\langle f_{1}\left(\tilde{x}_{1}, x_{2}^{m}\right)-f_{1}\left(\tilde{x}_{1}(0), x_{2}^{m}\right), x_{1}-\tilde{x}_{1}\right\rangle \\
& \leq\left\langle f_{1}\left(x_{1}, x_{2}\right)-f_{1}\left(\tilde{x}_{1}, x_{2}^{m}\right), x_{1}-\tilde{x}_{1}\right\rangle+\left\|f_{1}\left(\tilde{x}_{1}, x_{2}^{m}\right)-f_{1}\left(\tilde{x}_{1}(0), x_{2}^{m}\right)\right\|\left\|x_{1}-\tilde{x}_{1}\right\| \\
& \leq\left\langle f_{1}\left(x_{1}, x_{2}\right)-f_{1}\left(\tilde{x}_{1}, x_{2}^{m}\right), x_{1}-\tilde{x}_{1}\right\rangle+L_{1}\left\|\left(\begin{array}{c}
\tilde{x}_{1} \\
x_{2}^{m}
\end{array}\right)-\left(\begin{array}{c}
\tilde{x}_{1}(0) \\
x_{2}^{m}
\end{array}\right)\right\|\left\|x_{1}-\tilde{x}_{1}\right\| \\
& \leq \lambda_{1}\left\|x_{1}-\tilde{x}_{1}\right\|^{2}+\gamma_{1}\left\|x_{2}-x_{2}^{m}\right\|\left\|x_{1}-\tilde{x}_{1}\right\|+L_{1} t\left\|f_{1}\left(\tilde{x}_{1}(0), x_{2}^{m}\right)\right\|\left\|x_{1}-\tilde{x}_{1}\right\| \\
& \leq \lambda_{1}\left\|x_{1}-\tilde{x}_{1}\right\|^{2}+\left(\gamma_{1} \frac{\left|T_{2}\right|}{2}+C_{1} t\right)\left\|x_{1}-\tilde{x}_{1}\right\|
\end{aligned}
$$

where $\left|T_{2}\right|$ denotes the diameter of $T_{2}$. Using the fact that $\left\|x_{1}-\tilde{x}_{1}\right\| \leq \frac{1}{2}\left(\alpha \| x_{1}-\right.$ $\tilde{x}_{1} \|^{2}+\frac{1}{\alpha}$ ) for any $\alpha>0$, we can write three formulas following the sign of $\lambda_{1}$. 
- if $\lambda_{1}<0$, we can choose $\alpha=\frac{-\lambda_{1}}{C_{1} t+\gamma_{1}\left|T_{2}\right| / 2}$, and we get the differential inequality:

$$
\frac{d\left(\left\|x_{1}-\tilde{x}_{1}\right\|^{2}\right)}{d t} \leq \lambda_{1}\left\|x_{1}-\tilde{x}_{1}\right\|^{2}+\frac{C_{1}^{2}}{-\lambda_{1}} t^{2}+\frac{C_{1} \gamma_{1}\left|T_{2}\right|}{-\lambda_{1}} t+\frac{\gamma_{1}^{2}\left(\left|T_{2}\right| / 2\right)^{2}}{-\lambda_{1}}
$$

- if $\lambda_{1}>0$, we can choose $\alpha=\frac{\lambda_{1}}{C_{1} t+\gamma_{1}\left|T_{2}\right| / 2}$, and we get the differential inequality:

$$
\frac{d\left(\left\|x_{1}-\tilde{x}_{1}\right\|^{2}\right)}{d t} \leq 3 \lambda_{1}\left\|x_{1}-\tilde{x}_{1}\right\|^{2}+\frac{C_{1}^{2}}{\lambda_{1}} t^{2}+\frac{C_{1} \gamma_{1}\left|T_{2}\right|}{\lambda_{1}} t+\frac{\gamma_{1}^{2}\left(\left|T_{2}\right| / 2\right)^{2}}{\lambda_{1}}
$$

- if $\lambda_{1}=0$, we can choose $\alpha=\frac{1}{C_{1} t+\gamma_{1}\left|T_{2}\right| / 2}$, and we get the differential inequality:

$$
\frac{d\left(\left\|x_{1}-\tilde{x}_{1}\right\|^{2}\right)}{d t} \leq\left\|x_{1}-\tilde{x}_{1}\right\|^{2}+C_{1}^{2} t^{2}+C_{1} \gamma_{1}\left|T_{2}\right| t+\gamma_{1}^{2}\left(\left|T_{2}\right| / 2\right)^{2}
$$

In every case, the differential inequalities can be integrated to obtain the formulas of the theorem.

It then follows a distributed version of Corollary 2.

Corollary 3. Given a positive real $\delta$, consider two sets of points $\tilde{x}_{1}^{1}, \ldots, \tilde{x}_{m_{1}}^{1}$ and $\tilde{x}_{1}^{2}, \ldots, \tilde{x}_{m_{2}}^{2}$ such that all the balls $B\left(\tilde{x}_{i_{1}}^{1}, \delta\right)$ and $B\left(\tilde{x}_{i_{2}}^{2}, \delta\right)$, for $1 \leq i_{1} \leq m_{1}$ and $1 \leq i_{2} \leq m_{2}$, cover $R_{1}$ and $R_{2}$. Suppose that there exists patterns $\pi_{i_{1}}^{1}$ and $\pi_{i_{2}}^{2}$ of length $k_{i_{1}}$ and $k_{i_{2}}$ such that:

1. $B\left(\left(\tilde{x}_{i_{1}}^{1}\right)_{\pi_{i_{1}}^{1}}^{k^{\prime}}, \delta_{\pi_{i_{1}}^{1}}^{k^{\prime}}\right) \subseteq S_{1}$, for all $k^{\prime}=1, \ldots, k_{i_{1}}-1$

2. $B\left(\left(\tilde{x}_{i_{1}}^{1}\right)_{\pi_{i_{1}}^{1}}^{k_{i_{1}}}, \delta_{\pi_{i_{1}}^{1}}^{k_{i_{1}}}\right) \subseteq R_{1}$.

3. $\frac{d^{2}\left(\delta_{j_{1}}^{\prime}(t)\right)}{d t^{2}}>0$ with $j_{1}=\pi_{i_{1}}^{1}\left(k^{\prime}\right)$ and $\delta^{\prime}=\delta_{\pi_{i_{1}}^{1}}^{k^{\prime}-1}$, for all $k^{\prime} \in\left\{1, \ldots, k_{i_{1}}\right\}$ and $t \in[0, \tau]$.

1. $B\left(\left(\tilde{x}_{i_{2}}^{2}\right)_{\pi_{i_{2}}^{2}}^{k^{\prime}}, \delta_{\pi_{i_{2}}^{2}}^{k^{\prime}}\right) \subseteq S_{2}$, for all $k^{\prime}=1, \ldots, k_{i_{2}}-1$

2. $B\left(\left(\tilde{x}_{i_{2}}^{2}\right)_{\pi_{i_{2}}^{2}}^{k_{i_{2}}}, \delta_{\pi_{i_{2}}^{2}}^{k_{i_{2}}}\right) \subseteq R_{2}$.

3. $\frac{d^{2}\left(\delta_{j_{2}}^{\prime}(t)\right)}{d t^{2}}>0$ with $j_{2}=\pi_{i_{2}}^{2}\left(k^{\prime}\right)$ and $\delta^{\prime}=\delta_{\pi_{i_{2}}^{2}}^{k^{\prime}-1}$, for all $k^{\prime} \in\left\{1, \ldots, k_{i_{2}}\right\}$ and $t \in[0, \tau]$.

The above properties induce a distributed control $\sigma=\left(\sigma_{1}, \sigma_{2}\right)$ guaranteeing (non simultaneous) recurrence in $R$ and safety in $S$. I.e.

- if $x \in R$, then $\phi_{\sigma}(t ; x) \in S$ for all $t \geq 0$

- if $x \in R$, then $\phi_{\sigma}\left(k_{1} \tau ; x\right)_{\mid 1} \in R_{1}$ for some $k_{1} \in\left\{k_{i_{1}}, \ldots, k_{i_{m_{1}}}\right\}$, and symmetrically $\phi_{\sigma}\left(k_{2} \tau ; x\right)_{\mid 2} \in R_{2}$ for some $k_{2} \in\left\{k_{i_{2}}, \ldots, k_{i_{m_{2}}}\right\}$ 


\section{Application}

We demonstrate the feasibility of our approach on a (linearized) building ventilation application adapted from [15]. The system is a four-room apartment subject to heat transfer between the rooms, with the external environment and with the underfloor. The dynamics of the system is given by the following equation:

$$
\frac{d T_{i}}{d t}=\sum_{j \in \mathcal{N}^{*} \backslash\{i\}} a_{i j}\left(T_{j}-T_{i}\right)+c_{i} \max \left(0, \frac{V_{i}-V_{i}^{*}}{\bar{V}_{i}-V_{i}^{*}}\right)\left(T_{u}-T_{i}\right) .
$$

The state of the system is given by the temperatures in the rooms $T_{i}$, for $i \in$ $\mathcal{N}=\{1, \ldots, 4\}$. Room $i$ is subject to heat exchange with different entities stated by the indexes $\mathcal{N}^{*}=\{1,2,3,4, u, o, c\}$. The heat transfer between the rooms is given by the coefficients $a_{i j}$ for $i, j \in \mathcal{N}^{2}$, and the different perturbations are the following:

- The external environment: it has an effect on room $i$ with the coefficient $a_{i o}$ and the outside temperature $T_{o}$, set to $30^{\circ} \mathrm{C}$.

- The heat transfer through the ceiling: it has an effect on room $i$ with the coefficient $a_{i c}$ and the ceiling temperature $T_{c}$, set to $30^{\circ} \mathrm{C}$.

- The heat transfer with the underfloor: it is given by the coefficient $a_{i u}$ and the underfloor temperature $T_{u}$, set to $17^{\circ} C\left(T_{u}\right.$ is constant, regulated by a PID controller).

The control $V_{i}, i \in \mathcal{N}$, is applied through the term $c_{i} \max \left(0, \frac{V_{i}-V_{i}^{*}}{\bar{V}_{i}-V_{i}^{*}}\right)\left(T_{u}-T_{i}\right)$. A voltage $V_{i}$ is applied to force ventilation from the underfloor to room $i$, and the command of an underfloor fan is subject to a dry friction. Because we work in a switching control framework, $V_{i}$ can take only discrete values, which removes the problem of dealing with a "max" function in interval analysis. In the experiment, $V_{1}$ and $V_{4}$ can take the values $0 \mathrm{~V}$ or $3.5 \mathrm{~V}$, and $V_{2}$ and $V_{3}$ can take the values $0 \mathrm{~V}$ or $3 \mathrm{~V}$. This leads to a system of the form (8) with $\sigma(t) \in U=\{1, \ldots, 16\}$, the 16 switching modes corresponding to the different possible combinations of voltages $V_{i}$. The system can be decomposed in sub-systems of the form (9)-(10). The sampling period is $\tau=30 \mathrm{~s}$. The parameters $V_{i}^{*}, \bar{V}_{i}, a_{i j}, b_{i}, c_{i}$ are given in [15] and have been identified with a proper identification procedure detailed in $[16]$.

The main difficulty of this example is the large number of modes in the switching system, which induces a combinatorial issue. The centralized controller was obtained with 256 balls in 48 seconds, the distributed controller was obtained with $16+16$ balls in less than a second. In both cases, patterns of length 2 are used. A sub-sampling of $h=\tau / 20$ is required to obtain a controller with the centralized approach. For the distributed approach, no sub-sampling is required for the first sub-system, while the second one requires a sub-sampling of $h=\tau / 10$. Simulations of the centralized and distributed controllers are given in Figure 3, where the control objective is to stabilize the temperature in $[20,22]^{4}$ while never going out of $[19,23]^{4}$. 
Table 1: Numerical results for centralized four-room example.

\begin{tabular}{|c|c|}
\hline & Centralized \\
\hline$R$ & {$[20,22]^{4}$} \\
{$[\tau$} & $30,23]^{4}$ \\
\hline Time subsampling & \multicolumn{2}{|c|}{ Yes } \\
\hline Complete control & $\max _{j=1, \ldots, 16} \lambda_{j}=-6.30 \times 10^{-3}$ \\
\hline Error parameters & $\max _{j=1, \ldots, 16} C_{j}=4.18 \times 10^{-6}$ \\
\hline Number of balls/tiles & 256 \\
Pattern length & 2 \\
\hline CPU time & 48 seconds \\
\hline
\end{tabular}

Table 2: Numerical results for the distributed four-room example.

\begin{tabular}{|c|c|c|}
\hline & Sub-system 1 & Sub-system 2 \\
\hline$R$ & \multirow{2}{*}{\multicolumn{2}{|c|}{$\begin{array}{l}{[20,22]^{2} \times[20,22]^{2}} \\
{[19,23]^{2} \times[19,23]^{2}}\end{array}$}} \\
\hline$S$ & & \\
\hline$\tau$ & \multicolumn{2}{|c|}{30} \\
\hline Time subsampling & No & $\tau / 10$ \\
\hline Complete control & Yes & Yes \\
\hline Error parameters & $\max _{4} \lambda_{j_{1}}^{1}=-1.39 \times 10^{-3}$ & $\max _{j_{2}=1} \lambda_{j_{2}}^{2}=-1.42 \times 10^{-3}$ \\
\hline & $\max _{j_{1}=1, \ldots, 4} \gamma_{j_{1}}^{1}=1.79 \times 10^{-4}$ & $\max _{j_{2}=1, \ldots, 4} \gamma_{j_{2}}^{2}=2.47 \times 10^{-4}$ \\
\hline & $\max _{j_{1}=1, \ldots, 4} C_{j_{1}}^{1}=4.15 \times 10^{-4}$ & $\max _{j_{2}=1, \ldots, 4} C_{j_{2}}^{2}=5.75 \times 10^{-4}$ \\
\hline Number of balls/tiles & 16 & 16 \\
\hline Pattern length & 2 & 2 \\
\hline CPU time & $<1$ second & $<1$ second \\
\hline
\end{tabular}

\section{$5 \quad$ Final remarks and future work}

We have given a new distributed control synthesis method based on Euler's method. The method makes use of the notions of $\delta$-ISS-stability and ISS Lyapunov functions. From a certain point of view, this method is along the lines of [6] and [11] which are inspired by small-gain theorems of control theory (see, e.g., [9]). In the future, we plan to apply our distributed Euler-based method to significant examples such as the 11-room example treated in $[12,14]$.

\section{References}

[1] David Angeli. A Lyapunov approach to incremental stability. In Proc. of IEEE Conference on Decision and Control,, volume 3, pages 2947-2952, 2000.

[2] Eugene Asarin, Thao Dang, and Antoine Girard. Hybridization methods for the analysis of nonlinear systems. Acta Informatica, 43(7):451-476, 2007. 

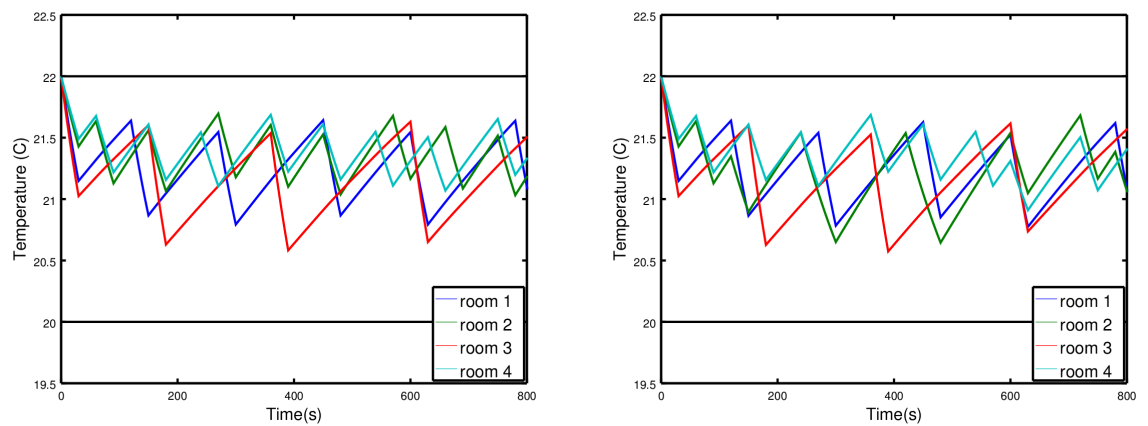

Figure 3: Simulation of the centralized (left) and distributed (right) controllers from the initial condition $(22,22,22,22)$.

[3] Kendall E Atkinson. An introduction to numerical analysis. John Wiley \& Sons, 2008.

[4] Xin Chen and Sriram Sankaranarayanan. Decomposed reachability analysis for nonlinear systems. In Proc. of IEEE Real-Time Systems Symposium, pages 13-24, 2016.

[5] Germund Dahlquist. Error analysis for a class of methods for stiff non-linear initial value problems. Numerical analysis, pages 60-72, 1976.

[6] Eric Dallal and Paulo Tabuada. On compositional symbolic controller synthesis inspired by small-gain theorems. In Proc. of IEEE Conference on Decision and Control, pages 6133-6138, 2015.

[7] Tzanko Donchev and Elza Farkhi. Stability and euler approximation of one-sided lipschitz differential inclusions. SIAM journal on control and optimization, 36(2):780-796, 1998.

[8] João P Hespanha, Daniel Liberzon, and Andrew R Teel. Lyapunov conditions for input-to-state stability of impulsive systems. Automatica, 44(11):2735-2744, 2008.

[9] Z-P Jiang, Andrew R Teel, and Laurent Praly. Small-gain theorem for ISS systems and applications. Mathematics of Control, Signals, and Systems, $7(2): 95-120,1994$.

[10] Zhong-Ping Jiang, Iven MY Mareels, and Yuan Wang. A Lyapunov formulation of the nonlinear small-gain theorem for interconnected iss systems. Automatica, 32(8):1211-1215, 1996.

[11] Eric S Kim, Murat Arcak, and Sanjit A Seshia. Compositional controller synthesis for vehicular traffic networks. In Proc. of IEEE Annual Conference on Decision and Control, pages 6165-6171, 2015.

[12] Kim G Larsen, Marius Mikučionis, Marco Muniz, Jiri Srba, and Jakob Haahr Taankvist. Online and compositional learning of controllers with application to floor heating. In Proc. of International Conference Tools and Algorithms for Construction and Analysis of Systems, 2016. 
[13] Adrien Le Coënt, Florian De Vuyst, Ludovic Chamoin, and Laurent Fribourg. Control synthesis of nonlinear sampled switched systems using Euler's method. In Proc. of International Workshop on Symbolic and Numerical Methods for Reachability Analysis, volume 247 of EPTCS, pages 18-33. Open Publishing Association, 2017.

[14] Adrien Le Coënt, Laurent Fribourg, Nicolas Markey, Florian De Vuyst, and Ludovic Chamoin. Distributed synthesis of state-dependent switching control. In International Workshop on Reachability Problems, pages 119133. Springer, 2016.

[15] Pierre-Jean Meyer. Invariance and symbolic control of cooperative systems for temperature regulation in intelligent buildings. Theses, Université Grenoble Alpes, September 2015.

[16] Pierre-Jean Meyer, Hosein Nazarpour, Antoine Girard, and Emmanuel Witrant. Experimental implementation of UFAD regulation based on robust controlled invariance. In Proc. of European Control Conference, pages 1468-1473, 2014.

[17] Guosong Yang and Daniel Liberzon. A lyapunov-based small-gain theorem for interconnected switched systems. Systems $\&$ Control Letters, 78:47-54, 2015. 